\title{
LOCALLY BOUNDED SPACES
}

\author{
by P. TH. LAMBRINOS \\ (Received 13th March 1974)
}

The three common definitions of local compactness require, respectively, each point to have a compact neighbourhood, a neighbourhood basis consisting of compact sets, or a closed compact neighbourhood. These definitions are equivalent in Hausdorff or in regular spaces but not in general $(3,7)$.

A. J. Ward has called a space quasi-locally compact (q.l.c.) if given any neighbourhood $N_{1}$ of any point $x$ of the space, there exists a neighbourhood $N_{2}$ of $x$ such that $N_{2} \subset N_{1}$ and $N_{2}$ is contained in some finite union of members of every open cover of $N_{1}(9)$.

Here, as indicated in a previous paper (5), we introduce a new class of spaces, called locally bounded (1.b.), strictly larger than the class of q.1.c. spaces, as well as that of locally compact (l.c.) spaces (in any of their three common definitions).

L.b. spaces having by their definition a simplicity advantage, at the same time preserve many properties of the l.c. spaces. We recall from (5), that a subset of a topological space $(X, \tau)$ is called bounded in $(X, \tau)$ if it is contained in some finite union of members of every open cover of the whole space $X .(X, \tau)$ is compact iff $X$ is bounded. Subsets of compact sets are bounded but in general there are bounded proper subsets of $X$ which are contained in no one compact set. Subsets, finite unions, continuous images and arbitrary products of bounded subsets are bounded.

Definition 1. A topological space is called locally bounded (1.b.) if each point has at least one bounded neighbourhood or, equivalently, if each point has a neighbourhood basis consisting of bounded subsets of the space.

Every compact space is l.b. Every regular non-l.c. space is not l.b. (see below). From the above we have

Proposition 1. Every l.c. or q.l.c. space is a l.b. space.

The converse of the above proposition does not hold in general. Indeed, the example below shows that there exist spaces (even Hausdorff spaces) 1.b. but which are not 1.c. or q.l.c.

Example 1. Let $X$ consist of points $a, b, c_{i}, a_{i j}, b_{i j}, i, j=1,2,3, \ldots$ and let $\tau$ be the topology on $X$ defined by the neighbourhood basis $\mathscr{B}(x)$ of each point $x \in X$ as follows

$$
\begin{aligned}
& \mathscr{B}(a)=\left\{V^{n}(a)=\{a\} \cup\left\{a_{i j}: i \geqq n, j \in \mathbb{N}\right\}: n \in \mathbb{N}\right\} \\
& \mathscr{B}(b)=\left\{V^{n}(b)=\{b\} \cup\left\{b_{i j}: i \geqq n, j \in \mathbb{N}\right\}: n \in \mathbb{N}\right\} \\
& \mathscr{B}\left(a_{i j}\right)=\left\{\left\{a_{i j}\right\}\right\}, \mathscr{B}\left(b_{i j}\right)=\left\{\left\{b_{i j}\right\}\right\}, \\
& \mathscr{B}\left(c_{i}\right)=\left\{V^{n}\left(c_{i}\right)=\left\{c_{i}\right\} \cup\left\{a_{i j}: j \geqq n\right\} \cup\left\{b_{i j}: j \geqq n\right\}: n \in \mathbb{N}\right\} \\
& \text { E.M.S. }-19 / 4-Y
\end{aligned}
$$


The above space has been constructed by P. Uryshon (see (1)) for a different purpose.

First we prove that a subset $S$ of $X$ is bounded in $(X, \tau)$ iff $S \cap C$ is finite where $C=\left\{c_{i}: i \in \mathbb{N}\right\}$. Indeed, if $S$ is bounded, then it must be contained in a finite union of members of the open cover

$$
\left\{V^{n}(a), V^{n}(b)\right\} \cup\left\{V^{n}\left(c_{i}\right),\left\{a_{i j}\right\},\left\{b_{i j}\right\}: i \in \mathbb{N}, j \in \mathbb{N}\right\}
$$

of $X$ each member of which contains at most one element of $C$. Conversely, let $S \cap C$ be finite and $\mathscr{Q}$ any open cover of $X$. Then, we take an open refinement $\left\{V^{n_{a}}(a), V^{n_{b}}(b)\right\} \cup\left\{V^{n_{i}}\left(c_{i}\right),\left\{a_{i j}\right\},\left\{b_{i j}\right\}: i \in \mathbb{N}, j \in \mathbb{N}\right\}$ of 2 . It follows that $S-\bigcup\left\{V^{n_{1}}\left(c_{i}\right): i \leqq \max \left\{n_{a}, n_{b}\right\}\right\} \cup V^{n_{a}}(a) \cup V^{n_{b}}(b)$ is a finite subset and thus, $S$ is contained in a finite union of members of $\mathcal{Q}$. Therefore, $(X, \tau)$ is 1.b. However, the Hausdorff space $(X, \tau)$ is neither l.c. nor q.l.c. Indeed, there exists the point $a$ and a neighbourhood $V^{n}(a)$ of $a$, such that for every basic neighbourhood $V^{m}(a) \subset V^{n}(a)$ there exists the open cover

$$
\left\{V^{m+1}(a)\right\} \cup\left\{\left\{a_{i j}\right\}: m \geqq i \geqq n, j \in \mathbb{N}\right\} \quad \text { of } V^{n}(a)
$$

which does not contain a finite subcover of $V^{m}(a)$.

Another example, showing that the class of $1 . b$. spaces contains strictly the class of l.c. spaces is the following space, described in $(8$, p. 88).

Example 2. If $R$ is the set of real numbers with the Euclidean topology and if $Q$ is the set of rationals, $\tau^{*}$ is defined to be the topology generated by all sets $\{x\} \cup(Q \cap U)$ where $x \in U \in \tau$. The Hausdorff space $\left(\mathrm{R}, \tau^{*}\right)$ is not regular and thus, it is not l.c. However, it is shown that $\left(R, \tau^{*}\right)$ is l.b. since a subset $S \subset R$ is bounded in $\left(R, \tau^{*}\right)$ iff it is contained in some bounded (in the usual order sense) interval and contains at most a finite number of irrationals.

Indeed, the bounded subset $S$ must be contained in some finite union of members of the open cover $\{\{x\} \cup[Q \cap(x-1, x+1)]: x \in R\}$ of $R$. Conversely, by essentially the same argument used to show that the interval $[a, b]$ is compact it can be shown that every subset of any bounded (in the usual order sense) interval of rational numbers is bounded in $\left(X, \tau^{*}\right)$, (see also (2, Ex. 1) where " absolutely bounded" coincides with " bounded").

A $T_{3}$ (not necessarily Hausdorff) space is l.b. iff it is l.c. This is shown in an essentially different terminology in (6).

Proposition 2. For every non-compact space the complement of the adherence of the filter of the complements of the bounded subsets of the space is the set of the points which belong to some bounded neighbourhood.

Corollary 1. A non-compact space is l.b. iff the filter of the complements of the bounded subsets of the space has empty adherence.

Proposition 3. The image of a l.b. space by an open continuous surjection is l.b.

Note that a continuous image of a l.b. space is not necessarily l.b., e.g. any non-1.b. space may be considered as the continuous image of the discrete topology on the same set, by making use of the identity function. 
Proposition 4. If a product space $(X, \tau)$ contains a non-empty open bounded subset, then the coordinate spaces $\left(X_{i}, \tau_{i}\right), i \in I$ are compact with the possible exception of at most a finite number of them.

Proof Let $\Pi V_{i}$ be a non-empty basic open bounded subset of $(X, \tau)$, where $V_{i}=X_{i}$ except for at most a finite number of indices $i \in F-I$. Since boundedness is projective (5, Cor. 3.1$),\left(X_{i}, \tau_{i}\right)$ is compact for each $i \in I-F$.

Theorem 1. A product space is l.b. iff all coordinate spaces are l.b. and at most a finite number of them are not compact.

Proof. Necessity follows from Propositions 3 and 4. Conversely, given a point $x \in X$, take $V_{i}=X_{i}$ for each compact coordinate space $\left(X_{i}, \tau_{i}\right)$ and $V_{i}$ a bounded in $\left(X_{i}, \tau_{i}\right)$ neighbourhood of $x_{i}$ for the remainder. Since boundedness is productive, $\Pi V_{i}$ is a bounded neighbourhood of $x$.

Proposition 5. Every closed subspace $\left(B, \tau_{B}\right)$ of a l.b. space $(X, \tau)$ is l.b.

Proof. For every bounded in $(X . \tau)$ neighbourhood $V$ of a point $x \in B$ the set $V \cap B$ is shown (by $(5, \S 2.4),(2, \S 2))$ to be bounded in $\left(B, \tau_{B}\right)$ because $\operatorname{cl}(V \cap B) \subset B$.

A non-closed subspace of a 1.b. space, is not necessarily 1.b., for example the set $Q$ of rationals with topology induced by the Euclidean topology on the set $R$ of reals.

An open subspace of a l.b. space is not necessarily l.b. Indeed, the subspace $A=\{a\} \cup\left\{a_{i j}: i, j \in \mathbb{N}\right\}$ of the space $X$ of Example 1 is open and $T_{3}$ since it is a bounded subset of $X(5, \S 2.8)$ and thus, 1.c. if it is 1.b. But there is not any compact neighbourhood of $a$ consisting of elements of $A$.

Theorem 2. The disjoint topological union $(X, \tau)$ of a family of spaces $\left(X_{i}, \tau_{i}\right), i \in I$ is l.b. iff each space $\left(X_{i}, \tau_{i}\right)$ is l.b.

In the case of Hausdorff spaces we can get as special cases of Propositions 4, 5 and of Theorems 1, 2 well-known results (e.g. see (4)) by replacing "bounded" by " compact".

Another generalisation of a known fact is given by

Proposition 6. Each compact subset of a l.b. space has a neighbourhood basis consisting of bounded subsets.

Proposition 7. Every Hausdorff l.b. space is locally regular but not necessarily regular.

Definition 2. A l.b. (resp. Hausdorff l.b.) space is called minimal l.b. (resp. minimal Hausdorff l.b.) if there is not any strictly weaker (i.e. coarser) l.b. (resp. Hausdorff 1.b.) topology on the same set.

A compact space is not necessarily minimal l.b. but a Hausdorff compact space is minimal Hausdorff and thus minimal Hausdorff l.b. 
Although the next theorem is not a generalisation of the corresponding theorem for Hausdorff l.c. spaces (1), their proofs are almost identical.

Theorem 3. If a minimal l.b. space $(X, \tau)$ contains at least one closed compact set $A$ having non empty interior then $(X, \tau)$ is a compact space.

Proof. Let $(X, \tau)$ be non compact, $x$ an interior point of $A$ and $\mathscr{G}$ the open filter basis of the complements of the closed compact sets. A new topology $\tau^{*}$ is constructed on $X$ by taking for each $y \neq x, \mathscr{B}^{*}(y)=\mathscr{B}(y)$, i.e. the same open neighbourhood basis as in $\tau$ and $\mathscr{B}^{*}(x)=\mathscr{B}(x) \cap \mathscr{G}$. The topology $\tau^{*}$ is strictly weaker than $\tau$ because the $\tau$-neighbourhood $A$ of $x$ is not a $\tau^{*}$-neighbourhood since otherwise $(X, \tau)$ would be compact. It is proved that $\left(X, \tau^{*}\right)$ is compact. Indeed, if a filter $\mathscr{F}$ on $X$ has an adherent point in $(X, \tau)$ it will have non empty adherence in $\left(X, \tau^{*}\right)$. Otherwise, the point at the infinity of the one-point compactification of $(X, \tau)$ will be an adherent point of $\mathscr{F}$. It follows, that for every $F \in \mathscr{F}$ and $G \in \mathscr{G}$ we have $F \cap G \neq \phi$ and thus, $x$ is an adherent point in $\left(X, \tau^{*}\right)$ of the filter $\mathscr{F}$.

Hence, $\left(X, \tau^{*}\right)$ is $1 . b$. and strictly weaker than $(X, \tau)$, contradicting the hypothesis. Therefore, $(X, \tau)$ is necessarily compact.

Added in proof: The author found that from a different point of view (that of the one point compactification) and in a different context (that of not necessarily topological, separated closure spaces) a similar definition to that of a locally bounded space has been given by L. Skula (Ordered set of classes of compactifications, Czechoslovak Math. J. 19 (94), 1969, 42-59) on p. 54, where "l-compact spaces" are defined.

\section{REFERENCES}

(1) M. P. Berri, Minimal topological spaces, Trans. Amer. Math. Soc. 108 (1963), 97-105.

(2) S. Gagola and M. Gemignani, Absolutely bounded sets, Math. Japan 13 (1968), 129-132.

(3) J. I. Gross, A Third definition of local compactness, Amer. Math. Monthly 74 (1967), 1120-1122. 1965).

(4) J. L. Kelley, General Topology (van Nostrand, New York, London, Toronto,

(5) P. Th. Lambrinos, A topological notion of boundedness, Manuscripta Math. 10 (1973), 289-296.

(6) N. Oeconomidis, Sur les ensembles bornés dans un espace topologique, $A n$. Fac. Phys. Math. Univ. Thessaloniki (to appear).

(7) P. S. Schnare, Two definitions of local compactness. Amer. Math. Monthly, 72 (1965), 764-765. 
(8) L. A. Steen and J. A. Seebach, Jr, Counterexamples in Topology (Holt, Rinehart, Winston, New York, 1970).

(9) A. J. WARD, Problem in "Proceedings of the international symposium on topology and its applications, Herceg-Novi, 1968", p. 352. (Savez Matematičara, Beograd, Yugoslavia, 1969).

\section{UNIVERSITY OF THESSALONIKI}

GREECE 